\title{
EDAD Y ANTIGÜEDAD LABORAL DE LOS PROFESIONALES DE ENFERMERÍA COMO ANTECEDENTES DEL COMPROMISO AFECTIVO EN LOS HOSPITALES PRIVADOS DE JALISCO, MÉXICO
}

AGE AND WORKING SENIORITY OF NURSING PROFESSIONALS AS BACKGROUND TO THE AFFECTIVE COMMITMENT IN THE PRIVATE HOSPITALS IN JALISCO, MEXICO

José Alejandro Jaime Vargas Posgrado en Negocios.

Universidad Autónoma de Guadalajara. Zapopan, Jalisco, (México). E-mail: jose.jaime@edu.uag.mx ORCID: https://orcid.org/0000-0002-4821-1136

Recepción: 26/09/2019 Aceptación: 06/11/2019 Publicación: 24/11/2020

Citación sugerida:

Jaime, J. A. (2020). Edad y antigüedad laboral de los profesionales de enfermería como antecedentes del compromiso afectivo en los hospitales privados de Jalisco, México. 3C Empresa. Investigación y pensamiento crítico, 9(4), 53-71. https:// doi.org/10.17993/3cemp.2020.090444.53-71 


\section{RESUMEN}

El Compromiso organizacional es uno de los constructos de estudio tanto de las ciencias sociales como económico administrativas, iniciando por los autores Meyer y Allen (1991), quienes consideraron que es importante conocer el sentir de las personas hacia su empresa propusieros tres dimensiones a investigar el compromiso afectivo, el normativo y de continuidad.

Para la realización de este trabajo se utilizó una muestra de 149 profesionales de la salud de Guadalajara y Zapopan, (Jalisco) para determinar los factores sociodemográficos de los participantes. Se utilizó el modelo del Compromiso Organizacional propuesto por Meyer y Allen (1991) y para los propósitos de este estudio se utilizó solamente la dimensión del compromiso afectivo, que tiene 6 ítems, con el objetivo de determinar si la edad y antigüedad laboral son determinantes o antecedentes de la permanencia en el trabajo.

Los resultados obtenidos muestran que la edad y la antigüedad laboral influyen en el compromiso afectivo de los profesionales de la salud, los resultados coinciden con los autores Dorien y Cols (2010) y Ng y Fieldman (2011) citados por Böhrt, Solares y Romero (2014), concuerdan que el compromiso afectivo es más bajo en empleados de mayor edad y de antigüedad laboral y es más débil o va disminuyendo a medida que aumentaban los años de antigüedad laboral, los resultados obtenidos en este estudio coinciden con los autores mencionados y se concluye que de los 149 profesionales de enfermería de Guadalajara y Zapopan, (Jalisco) a mayor edad y antigüedad laboral del profesional de enfermería en los hospitales privados de estudio de Guadalajara y Zapopan disminuye el compromiso afectivo. Otra de las conclusiones es que los profesionales de enfermería no serían felices en pasar el resto de su vida en la misma empresa.

\section{PALABRAS CLAVE}

Compromiso afectivo, Edad, Antigüedad laboral, Profesionales de la salud, Permanencia. 


\section{ABSTRACT}

Organizational commitment is one of the study constructs of both the social and administrative economic sciences, beginning with the authors Meyer and Allen (1991), who considered that it is important to know the feelings of people towards their company, proposing three dimensions to investigate the affective, normative and continuity commitment.

To carry out this work, a sample of 149 health professionals from Guadalajara and Zapopan, (Jalisco) was used to determine the sociodemographic factors of the participants. The Organizational Commitment model proposed by Meyer and Allen (1991) was used and for the purposes of this study only the affective commitment dimension, which has 6 items, was used in order to determine if age and work seniority are determining or not. history of permanence at work.

The results showed that age and work seniority influence the affective commitment of health professionals, the results coincide with the authors Dorien and Cols (2010) and Ng and Fieldman (2011) cited by Böhrt, Solares and Romero (2014), agree that affective commitment is lower in older and older employees and is weaker or decreases as the years of work seniority increased, the results obtained in this study coincide with the authors mentioned and it is concluded that of the 149 nursing professionals from Guadalajara and Zapopan, (falisco), the older and the older the nursing professional in the private study hospitals of Guadalajara and Zapopan decrease affective commitment. Another conclusion is that nursing professionals have not been happy to spend the rest of their lives in the same company.

\section{KEYWORDS}

Affective commitment, Age, Job tenure, Health professionals, Work permanence. 


\section{INTRODUCCIÓN}

La salud es uno de los derechos fundamentales para todo ser humano y es considerado un factor insustituible que influye en el bienestar social, el desarrollo, la economía y la educación de los pueblos, esto coincide con la Organización Mundial de la Salud (OMS) 2013 al considerar que son aspectos que obligan a las instituciones hospitalarias a ofrecer atención médica oportuna, aceptable, asequible y de calidad satisfactoria, así mismo, emitió la obligación que tienen las unidades médicas de asegurar una atención médica satisfactoria. La OMS plantea la necesidad por conocer aquellos factores organizacionales que originan las deficiencias existentes en las instituciones (públicas y privadas) de salud, por esa razón, es necesario tener información que permita a los hospitales atender las necesidades de su capital humano para disponer de programas de bienestar que busque elevar la satisfacción laboral y a su vez, el compromiso organizacional, y en consecuencia una mejor atención hospitalaria.

Un hospital es la célula fundamental de la prestación de los servicios de salud en cualquier sociedad y se comporta como una empresa productora de servicios hospitalarios, con una función de producción y una serie de entradas que son transformadas en el proceso, lo que da como resultado un producto hospitalario (Cortés-Martínez, 2010) y que tiene como objetivo responder a las necesidades y cuidados de la salud de los pacientes, atendiendo a las necesidades laborales de sus empleados y contribuir como empresa a la generación de resultados financieros. Dentro de los hospitales existen tienen diversos tipos de personal profesional como son doctores, especialistas, profesionales de la salud, administrativos, entre otros, Zurriaga et al. (2000) indican que los profesionales de organizaciones sanitarias han sido considerados como un grupo ocupacional que experimenta altos niveles de experiencias del trabajo de carácter negativo como ausentismo, abandono, estrés laboral, un estado de agotamiento físico, emocional o mental que puede tener consecuencias en la autoestima del empleado, esto puede ser causado por el entorno y ambiente laboral que incluye las actividades, puesto, responsabilidades, instalaciones, compañeros, supervisores, lo anterior puede incidir en las actitudes del empleado en su medio laboral. 
La generación de investigaciones relativas a variables como el clima organizacional, la satisfacción laboral, el compromiso organizacional, ha tenido gran importancia dentro de las organizaciones desde hace décadas, y del sector salud no ha sido la excepción, cada vez más, los hospitales públicos y privados a través de recursos humanos se han interesado en conocer la percepción de sus empleados sobre sus actividades, jefes, entorno y empresa para evaluar los resultados y tomar acciones orientadas a mejorar las áreas

Los primeros investigadores del compromiso organizativo (Becker, 1960; Kanter, 1968) citados por Iqbal (2008); identificaron que el compromiso es principalmente una función del comportamiento individual y la disposición de las personas para dar su energía a la organización a través de acciones y elecciones a lo largo del tiempo. Becker 1960; Kanter, 1968) citados por Iqbal (2008) 960; Kanter, 1968) citados por Iqbal (2008) 960; Kanter, 1968 citados por Iqbal (2008; 1960) describió el compromiso como la tendencia a participar en líneas de actividad consistentes como la intención de permanecer en la organización (Iqbal, 2008).

Al estudio del compromiso de los empleados se le unió el estudio de las organizaciones, de ahí se desprende las investigaciones sobre el compromiso organizacional, dos autores Meyer y Allen, entre otros autores, fueron los pioneros y realizaron estudios sobre el tema, para 1991 presentaron tres tipos de compromisos los cuales son el compromiso afectivo, compromiso el continuo y el compromiso normativo. Para este estudio se analiza solamente el compromiso afectivo porque este tipo de compromiso se ha asociado con resultados positivos como son una tasa de rotación más baja, absentismo, motivación, dedicación, etc. (Meyer y Allen, 1997), también se relaciona con la mejora de los aspectos operativos de la organización, como el aumento de los niveles de rendimiento, una mayor satisfacción laboral y una mayor participación (Khan y Zafar, 2013).

El compromiso afectivo tiene sus antecedentes en cuatro categorías: las características personales (edad, sexo, antigüedad laboral y educación) (Angle y Perry 1981; Glisson y Durick 1988, Morris y Sterers, 1977; Meyer y Allen, 1991) características estructurales (descentralización en la toma de decisiones, 
relación empleado/supervisor, claridad en los roles) Brooke, Russell y Price, 1988; Morris y Steers, 1980) citados por Meyer y Allen (1991) mencionan que las características relacionadas con el puesto y las experiencias (por experiencias se divide en dos categorías: aquellas que satisfacen las necesidades de los empleadores para sentirse cómodos en la organización, tanto físicas como psicológicas y aquellas que contribuyen a los sentimientos del empleado en su competencia en su rol en el trabajo) (Meyer y Allen, 1991).

\section{DESARROLLO}

\subsection{PLANTEAMIENTO DEL PROBLEMA}

El planteamiento del problema en este estudio consiste en examinar si la edad y la antigüedad laboral tienen una relación positiva con el compromiso afectivo, siendo ésta una variable del compromiso organizacional.

\subsection{OBJETIVO}

Determinar la relación entre la edad y la antigüedad laboral antecedentes o predecesores del compromiso afectivo así como su intención de permanencia en los hospitales privados de tercer nivel de atención en la ciudad de Guadalajara y Zapopan, Jalisco (México).

\subsection{HIPÓTESIS}

$\mathrm{H}_{1}$ : A mayor antigüedad laboral menor es el compromiso afectivo de los profesionales de enfermería. $\mathrm{H}_{2}$ : A mayor edad de los profesionales de enfermería menor es el compromiso afectivo

\subsection{PROBLEMA GENERAL}

¿ A mayor antigüedad laboral menor es el compromiso afectivo de los profesionales de enfermería? ¿A mayor edad de los profesionales de enfermería menor es el compromiso afectivo? 


\subsection{REVISIÓN DE LA LITERATURA}

En la revisión de la literatura existen estudios por Satarr y Jan (2015) citado por Böhrt, Solares y Romero (2014), quienes demuestran que hay una asociación considerable entre la satisfacción del trabajo, el compromiso organizacional y los atributos demográficos tales como género, edad, estado civil, experiencia, designación y calificación profesional de los empleados. Se han hecho estudios sobre la importancia de los factores sociodemográficos sobre el compromiso organizacional con resultados que señalan que la edad es una de las variables que más influyen en el compromiso afectivo tal como lo señala Baba y Jamal, estos autores en 1979 realizaron un estudio en Canadá con 377 empleados de diversas áreas de producción donde utilizaron seis variables entre ellas la edad, género, educación, estado civil, niños en casa e ingreso y en su conclusión determinaron que la edad era el único predictor demográfico del compromiso organizacional.

Por su parte Yucel y Erzincan en el año 2012 llevaron a cabo un estudio con maestros en varias escuelas de educación secundaria en Turquía analizando la satisfacción laboral y el compromiso organizacional, las variables de control estudiadas en este artículo son el género, la edad, el estado civil, el nivel educativo y la permanencia laboral, todas estas variables como antecedentes del compromiso organizativo y concluyeron que es especialmente la edad del empleado lo que afecta el compromiso organizativo y la satisfacción laboral. En su discusión postulan que los maestros más jóvenes, son más propensos a sentirse emocionalmente más apegados e identificados con los problemas de la organización como si fueran propios y expresaron el deseo de pasar el resto de su carrera en la organización mientras su satisfacción laboral sea alta o baja pero no moderada. Consideraron la edad en cinco categorías categorizando a los participantes por incrementos de 10 años (es decir, 21- 30, 31- 40, 41-50, 51- 60 y 61 y más).

Por su parte, Brimeyer, Perrucci y MacDermid, (2010), citados por Böhrt et al., (2014), analizaron la relación de las variables edad y antigüedad con el nivel de compromiso en una muestra de 400 empleados de una empresa manufacturera; los resultados mostraron que los trabajadores con mayor edad, sin importar su antigüedad en la organización, indicaban estar más comprometidos que los más 
jóvenes. Sin embargo, Dorien y cols. (2010) estudiaron la influencia de la edad en el desarrollo del compromiso de tipo Afectivo a partir de un meta-análisis de 83 estudios, revelando que el Compromiso Afectivo era más bajo en trabajadores con mayor edad, adicionalmente, establecieron que a mayor antigüedad en la organización, menor Compromiso Afectivo. Ng y Fieldman (2011) citados por Böhrt $e t$ al. (2014) analizaron esta misma relación y los resultados mostraron que a mayor antigüedad, más alto el compromiso; sin embargo el compromiso afectivo era más débil a medida que aumentaban los años de antigüedad (Böhrt et al., 2014).

También existen resultados opuestos entre las investigaciones para la edad y el vínculo de compromiso organizativo al mostrar que la edad tenía un vínculo significativamente positivo con el compromiso afectivo y normativo y una relación insignificante con el compromiso de continuación (Meyer y Allen, 1998) citado por Khan y Zafar. Se considera que los trabajadores de edad avanzada tengan un compromiso afectivo mayor debido a varias razones, incluyendo una mayor satisfacción con sus puestos de trabajo, mejores puestos, etc. La percepción del trabajo y la realización personal puede ser diferente para los empleados que se encuentran en diferentes grupos de edad. Los empleados en el rango de edad más joven pueden no desarrollar apego emocional a la organización en un corto período de tiempo y podrían mostrar menor disponibilidad para comprometerse con una organización por un mayor plazo (Khan y Zafar, 2013).

Investigaciones anteriores postulan que a medida que los trabajadores se quedan con una organización también es probable que aumente su nivel de compromiso (Mathieu y Zajac, 1990, citados Khan y Zafar, 2013). Por otro lado, Ng y Fieldman (2011) citados por Böhrt et al. (2014) mostraron también que a mayor antigüedad, más alto el compromiso; sin embargo el compromiso afectivo era más débil a medida que aumentaban los años de antigüedad en la organización (Böhrt et al., 2014). Un estudio hecho en Malasia realizado en empleados de "cuello blanco" encontró que el compromiso afectivo no estaba significativamente relacionado con la antigüedad laboral (Ahmad y Bakar, 2003) atribuyéndolo al incierto ambiente de negocios en el país. Otra investigación llevada a cabo por Nogueras (2006) citado 
por Khan y Zafar (2013) mostró un vínculo positivo entre los niveles de compromiso de las enfermeras a medida que aumentaba el número de años laborados en su organización (Khan y Zafar, 2013).

Por lo tanto, la edad y la antigüedad laboral fueron sugeridas por autores como Ritzer y Trice (1969), Alutto, Hrebiniak, y Alonso (1973), Meyer y Allen (1984) y Sheldon (1971) citados por Cohen (1993) como los mejores indicadores que generan la estabilidad laboral. Sin embargo, las conclusiones de la investigación han demostrado relaciones débiles entre el compromiso organizacional, la edad y la antigüedad laboral. Una explicación de estas débiles relaciones fue propuesta por Meyer y Allen (1984) argumentando que los empleados más jóvenes pueden estar más comprometidos debido a su conciencia de que, con menos experiencia trabajo, a menudo tienen menos oportunidades de trabajo (Cohen, 1993).

Los resultados demuestran que la edad y la antigüedad laboral influyen en el compromiso afectivo de los profesionales de la salud, los resultados coinciden con Dorien y Cols, (2010) al mencionar que el compromiso afectivo era más bajo en empleados de mayor edad, sin embargo, Ng y Fieldman (2011) citados por Böhrt et al. (2014) analizaron esta misma relación y los resultados mostraron que a mayor antigüedad, más alto el compromiso; sin embargo el compromiso afectivo era más débil a medida que aumentaban los años de antigüedad. De una población de 149 profesionales de la salud, 12 de ellos fueron quienes tenían más de 15 años trabajando en la misma organización, cuya edad estaba en el rango de 30 a 50 años.

\subsection{MARCO TEÓRICO}

Para los investigadores y personal de recursos humanos la importancia del compromiso organizacional se debe principalmente a que tiene un impacto directo en actitudes y conductas del empleado; como aceptación de metas, valores y cultura de la organización, menor ausentismo y baja rotación de personal. Por ello el compromiso organizacional es un concepto que ha crecido en importancia en la psicología organizacional, y se centra en estudiar los vínculos que se generan entre los empleados y la organización (Betanzos y Paz, 2007). 
Los primeros estudios sobre el compromiso organizacional data de los años sesentas y setentas del siglo pasado, autores como Porter, Steers, Mowda y Boulian dedicaron años en el estudio del compromiso, generando modelos teóricos e instrumentos que sentaron las bases de investigaciones posteriores. En los años ochenta surgen trabajos de autores como Allen y Meyer, en 1984 propusieron un modelo bidimensional para medir el compromiso organizacional en el que distinguían entre compromiso afectivo y de continuidad y en 1990 añadieron una tercera dimensión a su modelo a la que bautizaron como compromiso normativo (Gallardo, 2008).

El interés en un principio se orientó hacia resultados de la organización tales como el desempeño de los empleados, la eficacia, la satisfacción laboral, el absentismo entre otros. De las tres dimensiones, se considera para esta investigación analizar solamente el compromiso afectivo, este tipo de compromiso ocupa una posición vital, ya que capta el significado fundamental del compromiso, que es el apego emocional entre el empleado y la organización. El compromiso afectivo se define como "apego emocional del empleado a, identificación con y participación en la organización” (Meyer y Allen, 1991). en otras palabras, los empleados comprometidos afectivamente permanecen en el lugar de trabajo porque quieren (Kloutsiniotis y Mihail, 2017).

Estudios previos indicaron que en comparación con el compromiso de continuidad y el compromiso normativo, el compromiso afectivo se correlacionaba de una manera más significativa con los resultados del trabajo como el rendimiento, el absentismo (Meyer y Herscovitch, 2001). Mientras que otro estudio empírico demostró que el compromiso afectivo desempeñó un papel crucial, pero no completamente comprendido, tanto en la retención de empleados como en la promoción del bienestar del personal (Kloutsiniotis y Mihail, 2017).

\subsection{DISEÑO DEL ESTUDIO}

La presente investigación es exploratoria, el tipo de estudio es descriptivo. 


\subsubsection{METODOLOGÍA}

Se utilizó el instrumento del Copromiso Organizacional propuesto por Meyer y Allen (1991). El instrumento consta de 17 reactivos en total y solamente se utilizó para los propósitos de este estudio la dimensión compromiso afectivo que tiene 6 items.

1. Tengo una fuerte sensación de pertenecer a esta empresa;

2. Esta empresa tiene un gran significado para mí;

3. Me siento como parte de una familia en esta empresa;

4. Realmente siento como si los problemas de esta empresa fueran mis propios problemas;

5. Disfruto hablando de mi empresa con gente que no pertenece a ella;

6. Sería muy feliz pasando el resto de mi vida laboral en esta empresa.

Se utilizó una escala de Likert de 5 opciones (Muy en desacuerdo(1), Algo en desacuerdo (2), Ni de acuerdo ni en desacuerdo(3), Algo de acuerdo (5), Muy de acuerdo(6)).

La realización del análisis factorial y obtención de los resultados demográficos fue utiliando SPSS versión 23 para la obtención del KMO, prueba de Barlett y los resultados sociodemográficos de los participantes.

\subsection{UNIVERSO DE TRABAJO Y MUESTRA}

El universo de trabajo de la investigación fueron profesionales de enfermería de tres hospitales de Tercer Nivel de atención (espacialidades) ubicados en Guadalajara (Jalisco), y Zapopan (Jalisco).

Muestra: haciendo la limpieza de datos a través de SPSS con la técnica de Mahalanobis y de Alfa de Cronbach, de 170 instrumentos que se recibieron contestados en su totalidad, sólo 149 permanecieron para proceder a realizar el análisis, de los factores demográficos para este estudio se consideró la edad y años de antigüedad laboral del personal en las empresas. 
Se procedió a realizar la validación de criterio de las variables del estudio de la dimensión compromiso afectivo con la prueba de KMO y Barlett y los datos que se obtuvieron fueron:

Tabla 1. Prueba de KMO y Bartlett Compromiso Organizacional.

\begin{tabular}{|c|c|c|}
\hline \multicolumn{3}{|c|}{ Prueba de KMO y Bartlett emprendedurismo } \\
\hline Medida Kaiser-Meyer-Olkin de adecuación de muestreo & 866 \\
\hline \multirow{2}{*}{$\begin{array}{c}\text { Prueba de esfericidad de } \\
\text { Bartlett }\end{array}$} & Aprox. Chi-cuadrado & 322.54 \\
\hline & gl & 15 \\
\hline
\end{tabular}

Fuente: elaboración propia con resultados de SPSS.

\section{Distribución de puntuaciones del KMO y criterios de evaluación propuestos por Kaiser (1974). $1.00 \mathrm{KMO}>0.90$ Excelentes $\quad 0.90 \mathrm{KMO}>0.80$ Buenos $0.80 \mathrm{KMO}>0.70$ Aceptables $0.70 \mathrm{KMO}>0.60$ Mediocres $0.60 \mathrm{KMO}>0.50$ Malos $0.00 \mathrm{KMO} \leq 0.50$ Muy malos}

Fuente: (Frías-Navarro y Pascual, 2012).

En Tabla 1 se observa que se obtuvieron resultados del KMO 0.866, y de acuerdo a Frías-Navarro y Pascual (2012) es un valor bueno, así como la prueba de Barlett de 0.000 este valor es aceptable.

Tabla 2. Varianza Total Explicada.

\begin{tabular}{|c|c|c|c|}
\hline Componente & \multicolumn{3}{|c|}{ Autovalores iniciales } \\
\hline $\mathbf{1}$ & Total & \% de varianza & \% acumulado \\
\hline $\mathbf{2}$ & 3.427 & 57.120 & 57.120 \\
\hline $\mathbf{3}$ & 0.679 & 11.319 & 68.439 \\
\hline $\mathbf{4}$ & 0.612 & 10.193 & 78.631 \\
\hline $\mathbf{5}$ & 0.506 & 8.429 & 87.061 \\
\hline $\mathbf{6}$ & 0.399 & 6.653 & 93.714 \\
\hline
\end{tabular}

Fuente: elaboración propia con resultados de SPSS .

De acuerdo a la Tabla 2, la varianza total explicada indica que una sola pregunta responde el 57.12\% de toda la dimensión. 
Tabla 3. Tiempo en la empresa por Edad.

\begin{tabular}{|c|c|c|c|c|c|c|}
\hline \multicolumn{7}{|c|}{ Edad } \\
\hline \multicolumn{3}{|c|}{ Tiempo en la empresa } & Frecuencia & Porcentaje & $\begin{array}{l}\text { Porcentaje } \\
\text { válido }\end{array}$ & Porcentaje \\
\hline \multirow{4}{*}{$\begin{array}{c}\text { Menos de } 5 \\
\text { años }\end{array}$} & \multirow{4}{*}{ Válido } & $\begin{array}{c}\text { Menos de } 30 \\
\text { años }\end{array}$ & 74 & 76.3 & 76.3 & 76.3 \\
\hline & & $\begin{array}{c}\text { De } 30 \text { a } 50 \\
\text { años }\end{array}$ & 22 & 22.7 & 22.7 & 99.0 \\
\hline & & $\begin{array}{l}\text { Más de } 50 \\
\text { años }\end{array}$ & 1 & 1.0 & 1.0 & 100.0 \\
\hline & & Total & 97 & 100.0 & 100.0 & \\
\hline \multirow{4}{*}{ De 5 a 15 años } & \multirow{4}{*}{ Válido } & $\begin{array}{c}\text { Menos de } 30 \\
\text { años }\end{array}$ & 4 & 10.0 & 10.0 & 10.0 \\
\hline & & $\begin{array}{c}\text { De } 30 \text { a } 50 \\
\text { años }\end{array}$ & 34 & 85.0 & 85.0 & 95.0 \\
\hline & & $\begin{array}{c}\text { Más de } 50 \\
\text { años }\end{array}$ & 2 & 5.0 & 5.0 & 100.0 \\
\hline & & Total & 40 & 100.0 & 100.0 & \\
\hline $\begin{array}{l}\text { Más de } 15 \\
\text { años }\end{array}$ & Válido & $\begin{array}{c}\text { De } 30 \text { a } 50 \\
\text { años }\end{array}$ & 12 & 100.0 & 100.0 & 100.0 \\
\hline
\end{tabular}

Fuente: elaboración propia con resultados de SPSS.

\section{RESULTADOS}

La Tabla 3 muestra que las personas que tienen menos de 5 años trabajando en la empresa tienen menos de 30 años de edad (lo que representa el 76.3\% de las 97 que están en este rango), pero en el rango de antigüedad laboral de 5 a 15 años, este mismo grupo disminuye drásticamente a 4 trabajadores (lo que representa el 10\%). Por otro lado, este mismo grupo tiene el mayor porcentaje de trabajadores por antigüedad laboral en el rango de 30 a 50 años con 34 trabajadores (lo que representa el el 85\% de las 40 trabajadores), lo que signnifica que en este rango la estabilidad laboral es más relevante porque hay más permanencia. 
El contraste significativo lo prepresenta los profesionales de la salud que tienen más de 15 años de antigüedad laboral porque sólo 12 trabajadores en total. Por lo tanto, se confirma la Hipótesis $\left(\mathbf{H}_{1}\right)$ "a mayor es la antigüedad laboral menor es el compromiso afectivo" y "a mayor es la edad de los profesionales de enfermería menor es el compromiso afectivo" lo que confirma la Hipótesis $\left(\mathrm{H}_{2}\right)$.

Tabla 4. Resultados por edad.

\begin{tabular}{|c|c|}
\hline \multicolumn{2}{|c|}{ Edad=Menos de 30} \\
Matriz de componente a,b \\
\hline $\begin{array}{c}\text { 2. Esta empresa tiene un gran significado } \\
\text { personal para mí }\end{array}$ & $\mathbf{1}$ \\
\hline $\begin{array}{c}\text { 4. Realmente siento como si los problemas } \\
\text { de esta empresa fueran mis propios } \\
\text { problemas }\end{array}$ & 0.777 \\
\hline $\begin{array}{c}\text { 3. Me siento como parte de una familia en } \\
\text { esta empresa }\end{array}$ & 0.769 \\
\hline $\begin{array}{c}\text { 5. Disfruto hablando de mi empresa con } \\
\text { gente que no pertenece a ella }\end{array}$ & 0.736 \\
\hline $\begin{array}{c}\text { 1. Tengo una fuerte sensación de } \\
\text { pertenecer a mi empresa }\end{array}$ & 0.679 \\
\hline $\begin{array}{c}\text { 6. Sería muy feliz pasando el resto de mi } \\
\text { vida laboral en esta empresa }\end{array}$ & 0.678 \\
\hline $\begin{array}{c}\text { Método de extracción: análisis de componentes principales } \\
\text { a. Edad=Menos de } 30 \text { años } \\
\text { b. 1 componente extraído }\end{array}$ \\
\hline Fuente: elaboracion ropia con resultados de SPSS.
\end{tabular}

\begin{tabular}{|c|c|}
\hline \multicolumn{2}{|l|}{ Edad= De 30 a 50 años } \\
\hline \multicolumn{2}{|l|}{ Matriz de componente $a, b$} \\
\hline & Componente \\
\hline & 1 \\
\hline $\begin{array}{l}\text { 1. Tengo una fuerte sensación de } \\
\text { pertenecer a mi empresa }\end{array}$ & 0.867 \\
\hline $\begin{array}{l}\text { 2. Esta empresa tiene un gran significado } \\
\text { personal para mí }\end{array}$ & 0.825 \\
\hline $\begin{array}{l}\text { 3. Me siento como parte de una familia en } \\
\text { esta empresa }\end{array}$ & 0.776 \\
\hline $\begin{array}{l}\text { 5. Disfruto hablando de mi empresa con } \\
\text { gente que no pertenece a ella }\end{array}$ & 0.771 \\
\hline $\begin{array}{l}\text { 6. Sería muy feliz pasando el resto de mi } \\
\text { vida laboral en esta empresa }\end{array}$ & 0.738 \\
\hline $\begin{array}{l}\text { 4. Realmente siento como si los problemas } \\
\text { de esta empresa fueran mis propios } \\
\text { problemas }\end{array}$ & 0.650 \\
\hline \multicolumn{2}{|c|}{ Método de extracción: análisis de componentes principales } \\
\hline \multicolumn{2}{|l|}{ a. Edad=De 30 a 50 años } \\
\hline b. 1 componente extraído & \\
\hline
\end{tabular}

Fuente: elaboración propia con resultados de SPSS.

El presente estudio pretende analizar también la intención de permanencia del empleado en su empresa, los resutados que muestra la Tabla 4 del ítem: "Sería muy feliz pasando el resto de mi vida laboral en esta empresa" en este estudio se refiere a la intención de permanencia del empleado en su empresa/ organización. De acuerdo a los resultados se observa que a más años que el empleado tenga trabajando en su empresa sería feliz pasar el resto de su vida laboral en la misma organización. Sumando los valores 
"De acuerdo" y "Totalmente de acuerdo" los datos son: Menos de 5 años de antigüedad el 29.8\% de los empleados dicen estar felices de permanecer en la empresa el resto de su vida laboral. Entre 5 y 15 años representan el 60\% y con más de 15 años de antigüedad con el 75\%. Este resultado coincide con el presentado por Contreras y Contreras (2018) quienes presentan que un 90.1\% de los colaboradores tienen la intención de permanecer en la institución.

En la edad aquellos que tienen menos de 30 años y con una edad que oscila entre los de 30 a 50 años el resultado que muestra la Tabla 4 presenta en los últimos lugares de las seis preguntas que el empleado sería feliz en pasar el resto de su vida en la empresa. Este resultado coincide con la hipótesis $\left(\mathrm{H}_{1)}\right.$ y la hipótesis $\left(\mathrm{H}_{2}\right)$.

$\mathrm{H}_{1}$ : A mayor antigüedad laboral menor es el compromiso afectivo de los profesionales de enfermería. $\mathrm{H}_{2}$ : A mayor edad de los profesionales de enfermería menor es el compromiso afectivo.

\section{CONCLUSIONES}

Los resultados obtenidos muestran que la edad y la antigüedad laboral influyen en el compromiso afectivo de los profesionales de la salud, los resultados coinciden con los autores Dorien y Cols (2010) y Ng y Fieldman (2011) citados por Böhrt et al. (2014) concuerdan que el compromiso afectivo es más bajo en empleados de mayor edad y de antigüedad laboral y es más débil o va disminuyendo a medida que aumentaban los años de antigüedad laboral, los resultados obtenidos en este estudio coinciden con los autores mencionados y se concluye que de los 149 profesionales de enfermería de Guadalajara y Zapopan, (Jalisco) a mayor edad y antigüedad laboral del profesional de enfermería en los hospitales privados de estudio de Guadalajara y Zapopan disminuye el compromiso afectivo. Otra de las conclusiones es que los profesionales de enfermería no serían felices en pasar el resto de su vida en la misma empresa.

Las limitaciones de este estudio es que se basó solamente en los profesionales de enfermería en los municipios de Guadalajara y Zapopan, (Jalisco), con dos variables edad y antigüedad laboral con el 
compromiso afectivo, en una población de 149 empleados de hospitales privados. La recomendación es el estudio del compromiso normativo y de continuidad con factores de permanencia en los hospitales privados de Zapopan y Guadalajara, Jalisco (México) y se utilicen otras variables sociodemográficas.

\section{REFERENCIAS BIBLIOGRÁFICAS}

Baba, V. V., y Jamal, M. (1979). On Becker's Theory of Commitment: An Empirical Verification Among Blue-Collar Workers. Relations industrielles / Industrial Relations, 34(1), 123-139. https:// www.riir.ulaval.ca/sites/riir.ulaval.ca/files/1979_34-1_7.pdf

Betanzos, N., y Paz, F. (2007). Análisis psicométrico del compromiso organizacional como variable actitudinal. Anales de psicología, 23(2), 207-215. https://www.redalyc.org/pdf/167/16723205.pdf

Böhrt, R., Solares, L., y Romero, G. (2014). Evolución del contrato psicológico y el compromiso organizacional con la edady la antigüedad. Ajayu. Órgano de Difusión Científica del Departamento de Psicología de la Universidad Católica Boliviana “San Pablo". https: / / www.redalyc.org/pdf/4615/461545457001. pdf

Gohen, A. (1993). Age and Job tenure in Relation to Organizational Commitment: A Meta-Analysis. Department of Political Science, University of Haifa.

Contreras, I. A., y Contreras, F. A. (2018). Compromiso organizacional y la intención de permanencia de los profesionales de enfermería en una institución de salud privada, Lima. Revista Científica De Ciencias De La Salud, 11(2). https://doi.org/10.17162/rccs.v1 1i2.1104

Frías-Navarro, D., y Pascual, M. (2012). Prácticas del análisis factorial exploratorio (AFE) en la investigación sobre conducta del consumidor. Suma Psicológica, 19(1), 47-58. https://www. uv.es/ friasnav/FriasNavarroMarcopsSoler.pdf 
Gallardo, E. (2008). Evolución en el estudio y medida del compromiso organizativo. Problemáticas y soluciones (Tesis Doctoral). Universidad de Barcelona (España). https://dialnet.unirioja.es/servlet/ articulo? codigo $=2734826$

González, V., López, J. R., Espejo, B., Zornoza, A., y Zurriaga, R. (2000). Efecto de las características del puesto de trabajo sobre la satisfacción, el compromiso y el absentismo en organizaciones sanitarias. Revista de Psicología Aplicada, 10(3). https://dialnet.unirioja.es/servlet/ articulo? codigo $=159342$

Iqbal, A. (2008). Organizational climate and employees' commitment: a study of the pakistani knitwear industry. Prince Sultan University.

Kaiser, H. F. (1974). An index of factorial simplicity. Psychometrika, 39, 31-36. https://doi.org/10.1007/ BF02291575

Khan, F., y Zafar, S. (2013). An Empirical Study of Affective Commitment across. Demographic groups in the Banking Sector of Pakistan. Pakistan Fournal of Commerce and Social Sciences, 7(3), 555563. http://www.jespk.net/publications/145.pdf

Kloutsiniotis, P. V., y Mihail, D. M. (2017). Linking innovative human resource practices, employee attitudes and intention to leave in healthcare services. Employee Relations, 39(1), 34-53. https://doi. org/10.1108/ER-11-2015-0205

Meyer, J. P., y Allen, N. J. (1984). Testing the 'side-bet theory' of organizational commitment: some methodological considerations. Fournal of Applied Psychology, 69(3), 372-378. https://doi. org/10.1037/0021-9010.69.3.372

Meyer,J. P., y Allen, N. J. (1991). A three component conceptualization of organizational commitment. Human Resource Management Review, 1, 61-98. https://doi.org/10.1016/1053-4822(91)90011-Z 


\section{Organización Mundial de la Salud (OMS). http://www.who.int/es}

Organización Panamericana de la Salud (OPS). https: / /www.paho.org/es

Yucel, I., y Erzincan, G. (2012). Job satisfaction, organizational commitment and demographic characteristics among teachers in Turkey: Kounger is better? Procedia - Social and Behavioral Sciences, 46, 1598-1608. https://www.researchgate.net/publication/275541964_Job_Satisfaction_Organizational_ Commitment_and_Demographic_Characteristics_Among_Teachers_in_Turkey_Younger_is_ Better/fulltext/5551595008ae956a5d25f455/Job-Satisfaction-Organizational-Commitmentand-Demographic-Characteristics-Among-Teachers-in-Turkey-Younger-is-Better.pdf 
